\title{
An iterative method for impulse noise removal
}

\author{
Xuesha $\mathrm{Wu}^{\mathrm{a}, *}$, Yun $\mathrm{Ji}^{\mathrm{b}}$ \\ a School of Liberal and International Education, Chongqing College of Electronic Engineering, \\ Chongqing 401331 China \\ b School of Electronics and Internet of Things, Chongqing College of Electronic Engineering, \\ Chongqing 401331 China
}

*Corresponding author, e-mail: wuxuesha2013@126.com

Received 31 Oct 2018

Accepted 28 Feb 2019

\begin{abstract}
Based on the conjugate gradient method, we propose a modified iterative method (MCG) for impulse noise removal based on the famous two-phase scheme. The noise candidates are firstly identified by the adaptive median filter, and then the MCG method recovers these noise candidates by minimizing an edge-preserving regularization function. A nice property of the MCG method is that the global convergence can be established without assuming that the objective function satisfies strong convexity under the Armijo-type line search. Numerical experiments show that the MCG method is effective to remove the impulse noise.
\end{abstract}

KEYWORDS: two-phase method, optimization, conjugate gradient method

MSC2010: 90C30 65K05

\section{INTRODUCTION}

In practice, images are often corrupted by impulse noise during image acquisition and transmission. Thus some algorithms for removing impulse noise have been intensively investigated. For example, the median filter and some of its variants ${ }^{1,2}$ have been constructed based on the nonlinear digital filters $^{3}$. These methods firstly locate possible noisy candidates, and then replace them with medians or their variants. Furthermore, these methods can detect the noisy pixels even at a high noise level. It is a pity that they cannot restore such pixels satisfactorily because they do not take into account local image features such as the possible presence of the edges. Subsequently, Nikolova ${ }^{4}$ proposed a variational method for impulse noise removal, which can preserve details and the edges well, but the grey level of every pixel is changed including uncorrupted ones. Other types of methods have also been extensively studied ${ }^{5-11}$.

In this paper we focus on the famous twophase method for impulse noise removal proposed by Chan et $\mathrm{al}^{11}$. The noisy candidates are firstly detected by the adaptive median filter ${ }^{1}$ or the adaptive centre-weighted median filter ${ }^{12}$ for different types of noise, and then the detected noisy pixels are recovered by minimizing an objective function by some optimization methods. Chan et al gave some numerical results to show that the two-phase method is powerful even for a salt-and-pepper noise ratio as high as $90 \%$. However, the given objective function is not smooth because it includes an $\ell_{1}$ data-fitting term. This implies that it is costly to obtain the minimizer in the second phase. To reduce the cost of computing, Chan et $\mathrm{al}^{13}$ showed that the non-smooth $\ell_{1}$ data-fitting term can be deleted from the objective function, because only detected noisy pixels are restored in the minimizing process. Furthermore, some experiments show that the quality of the recovered images is not affected. Thus the objective function proposed in Ref. 11 can be reduced to the following function. Let $X$ be the true image with $M$-by- $N$ pixels, and $\mathscr{A}=$ $\{1,2,3, \ldots, M\} \times\{1,2,3, \ldots, N\}$ be the index set of $X . \mathscr{N} \subset \mathscr{A}$ denotes the set of indices of the detected noisy pixels in the first phase. In the second phase, the detected noisy pixels can be restored by minimizing the following function.

$$
\begin{array}{r}
\mathscr{F}_{\alpha}(u)=\sum_{(i, j) \in \mathcal{N}}\left\{\sum_{(m, n) \in \mathscr{Y}_{i, j} \backslash \mathcal{N}} \varphi_{\alpha}\left(u_{i, j}-y_{m, n}\right)\right. \\
\left.+\frac{1}{2} \sum_{(m, n) \in \mathscr{Y}_{i, j} \cap \mathcal{N}} \varphi_{\alpha}\left(u_{i, j}-u_{m, n}\right)\right\},
\end{array}
$$

where $\mathscr{V}_{i, j}$ is the set of the four closest neighbours of the pixels at the position $(i, j) \in \mathscr{A}, y_{m, n}$ is the 
observed pixel value of the image at the position $(m, n), u=\left[u_{i, j}\right]_{(i, j) \in \mathscr{N}}$ is a column vector of the length $c$ ordered lexicographically in which $c$ is the number of elements of $\mathscr{N}$, and $\varphi_{\alpha}(t)$ is an edgepreserving function which has a great influence on the features of $\mathscr{F}_{\alpha}(u)$.

The objective function $\mathscr{F}_{\alpha}$ is smooth if the selected edge-preserving function $\varphi_{\alpha}$ is smooth. This means that some first-order optimization algorithms can be used to recover the corrupted images by minimizing (1). Cai et $\mathrm{al}^{14}$ used the conjugate gradient methods to recover the corrupted images without any line search. Yu et $\mathrm{al}^{15}$ proposed a descent spectral conjugate gradient method for impulse noise removal. A favourite property of the proposed method is that the search direction generated is a descent direction at each iteration. Under the strong Wolfe line search, its global convergence could be established if $\mathscr{F}_{\alpha}$ is strongly convex. Liu et $\mathrm{al}^{16}$ constructed a spectral gradient (SP) method to remove the impulse noise. The search direction generated by the SP method satisfies the sufficient descent property at each iteration, which is independent of any line search. Under the Armijo-type line search, the global convergence of the SP method is established for general smooth functions.

Hager et al ${ }^{17}$ proposed a nonlinear conjugate gradient method where the directions are generated by the rule

$$
d_{0}=-g_{0}, \quad d_{k}=-g_{k}+\beta_{k}^{\mathrm{HZ}} d_{k-1}, \quad k \geqslant 1,
$$

with

$$
\beta_{k}^{\mathrm{HZ}}=\frac{1}{d_{k-1}^{\mathrm{T}} y_{k-1}}\left(y_{k-1}-2 d_{k-1} \frac{\left\|y_{k-1}\right\|^{2}}{d_{k-1}^{\mathrm{T}} y_{k-1}}\right)^{\mathrm{T}} g_{k} .
$$

Under the standard Wolfe line search, the global convergence of this method is established for strongly convex smooth functions. To prove the global convergence for general smooth functions, they set $\beta_{k}^{\mathrm{CG}}=\max \left\{\beta_{k}^{\mathrm{HZ}}, \eta_{k}\right\}$, where

$$
\eta_{k}=\frac{-1}{\left\|d_{k-1}\right\| \min \left\{\eta,\left\|g_{k-1}\right\|\right\}}, \quad \eta>0 .
$$

This is the famous CG_DESCENT method, which is one of the most efficient conjugate gradient methods for solving unconstrained optimization problems. In this study, we are interested in the parameter $\beta_{k}^{\mathrm{HZ}}$. Based on the parameter $\beta_{k}^{\mathrm{HZ}}$, we propose a modified conjugate gradient (MCG) method for impulse noise removal. The MCG method inherits the nice property of the $\mathrm{HZ}$ method, i.e., the search direction generated by the MCG method always satisfies the sufficient descent property independent of any line search. In particular, its global convergence can be established for general smooth functions under the Armijo-type line search. Numerical experiments show that the proposed MCG method performs well for impulse noise removal.

\section{ALGORITHM AND ITS GLOBAL CONVERGENCE}

We start with the convergence theorem for general smooth function $f(x)$. Firstly, we give the modified conjugate gradient method.

\section{Algorithm 1}

Step 1: Give $x_{0} \in \mathbb{R}^{n}, \rho \in(0,1), \tau>0, \delta \in(0,1)$, $t>1 / 4, \varepsilon>0$. Set $k=0$.

Step 2: If $\left\|g_{k}\right\| \leqslant \varepsilon$, stop.

Step 3: Define $d_{k}$ by

$$
d_{k}= \begin{cases}-g_{k}, & k=0, \\ -g_{k}+\beta_{k} d_{k-1}, & k \geqslant 1,\end{cases}
$$

where $g_{k}$ denotes the gradient of the objective function at the $k$ th iterate and

$$
\beta_{k}=\frac{g_{k}^{\mathrm{T}} y_{k-1}}{d_{k-1}^{\mathrm{T}} \gamma_{k-1}}-\frac{t\left\|y_{k-1}\right\|^{2}}{\left(d_{k-1}^{\mathrm{T}} \gamma_{k-1}\right)^{2}} g_{k}^{\mathrm{T}} d_{k-1},
$$

$$
\gamma_{k-1}=y_{k-1}+r_{k-1} d_{k-1}, y_{k-1}=g_{k}-g_{k-1}, r_{k-1}=
$$$$
1+\max \left\{0,-d_{k-1}^{\mathrm{T}} y_{k-1} / d_{k-1}^{\mathrm{T}} d_{k-1}\right\}
$$

Step 4: Compute the step-size $\alpha_{k}$.

Step 5: Let $x_{k+1}=x_{k}+\alpha_{k} d_{k}$, set $k:=k+1$, go to Step 2.

The following result indicates that the MCG method must satisfy the sufficient descent condition without any line search.

Lemma 1 Let the sequences $\left\{d_{k}\right\}$ and $\left\{g_{k}\right\}$ be generated by the MCG method. Then

$$
g_{k}^{\mathrm{T}} d_{k} \leqslant-\left(1-\frac{1}{4 t}\right)\left\|g_{k}\right\|^{2}, k \geqslant 0 .
$$

Proof: (4) holds for $k=0$. For $k \geqslant 1$, from (2) we have

$$
\begin{array}{r}
g_{k}^{T} d_{k}=-\left\|g_{k}\right\|+\frac{\left(g_{k}^{T} y_{k-1}\right)\left(g_{k}^{T} d_{k-1}\right)}{d_{k-1}^{T} \gamma_{k-1}} \\
-\frac{t\left\|y_{k-1}\right\|^{2}\left(g_{k}^{T} d_{k-1}\right)^{2}}{\left(d_{k-1}^{T} \gamma_{k-1}\right)^{2}}
\end{array}
$$




$$
\begin{aligned}
=[ & -\left\|g_{k}\right\|\left(d_{k-1}^{T} \gamma_{k-1}\right)^{2} \\
& +\frac{d_{k-1}^{T} \gamma_{k-1}}{\sqrt{2 t}} g_{k}^{T}\left(\sqrt{2 t} g_{k}^{T} d_{k-1}\right) y_{k-1} \\
& \left.\quad-t\left\|y_{k-1}\right\|^{2}\left(g_{k}^{T} d_{k-1}\right)^{2}\right] /\left(d_{k-1}^{T} \gamma_{k-1}\right)^{2} \\
\leqslant & {\left[-\left\|g_{k}\right\|\left(d_{k-1}^{T} \gamma_{k-1}\right)^{2}+\frac{\left(d_{k-1}^{T} \gamma_{k-1}\right)^{2}}{4 t}\left\|g_{k}\right\|^{2}\right.} \\
& +t\left(g_{k}^{T} d_{k-1}\right)^{2}\left\|y_{k-1}\right\|^{2} \\
& \left.-t\left\|y_{k-1}\right\|^{2}\left(g_{k}^{T} d_{k-1}\right)^{2}\right] /\left(d_{k-1}^{T} \gamma_{k-1}\right)^{2} \\
= & -\left(1-\frac{1}{4 t}\right)\left\|g_{k}\right\|^{2} .
\end{aligned}
$$

To prove the global convergence of the MCG method, the following Armijo line search is needed, i.e., the step-size $\alpha_{k}=\max \left\{\rho^{i} \tau\left|g_{k}^{\mathrm{T}} d_{k}\right| /\left\|d_{k}\right\|^{2}, i=\right.$ $0,1,2,3, \ldots\}$ satisfies

$$
f\left(x_{k}+\alpha_{k} d_{k}\right) \leqslant f\left(x_{k}\right)-\delta \alpha_{k}^{2}\left\|d_{k}\right\|^{2}, \quad k \geqslant 0 .
$$

The following result is easily obtained under the given line search.

Lemma 2 Assume that the level set $\Gamma=\left\{x \in \mathbb{R}^{n} \mid\right.$ $\left.f(x) \leqslant f\left(x_{0}\right)\right\}$ is bounded. Let the sequence $\left\{d_{k}\right\}$, $k \geqslant 0$, be generated by the MCG method, and the stepsize $\alpha_{k}$ satisfies (5). Then

$$
\lim _{i \rightarrow \infty} \alpha_{i}\left\|d_{i}\right\|=0 .
$$

Proof: By (5), we have

$$
\sum_{i=0}^{k} \delta \alpha_{i}^{2}\left\|d_{i}\right\|^{2} \leqslant f\left(x_{0}\right)-f\left(x_{k+1}\right) .
$$

Since the level set $\Gamma$ is bounded, then

$$
\sum_{i=0}^{\infty} \delta \alpha_{i}^{2}\left\|d_{i}\right\|^{2}<\infty
$$

This indicates that the conclusion (6) holds.

Theorem 1 Assume that the level set $\Gamma=\left\{x \in \mathbb{R}^{n} \mid\right.$ $\left.f(x) \leqslant f\left(x_{0}\right)\right\}$ is bounded. Let the sequence $\left\{g_{k}\right\}, k \geqslant$ 0 , be generated by the MCG method, and the step-size $\alpha_{k}$ satisfies (5). If $g$ satisfies the Lipschitz condition, i.e., there exists a constant $L>0$ such that

$$
\|g(x)-g(y)\| \leqslant L\|x-y\|, \quad \forall x, y \in \Gamma,
$$

then we have

$$
\liminf _{k \rightarrow \infty}\left\|g_{k}\right\|=0
$$

Proof: From the definition $\gamma_{k-1}$ in the MCG algorithm, we have

$$
\begin{aligned}
d_{k-1}^{\mathrm{T}} \gamma_{k-1} & =d_{k-1}^{\mathrm{T}} y_{k-1}+r_{k-1} d_{k-1}^{\mathrm{T}} d_{k-1} \\
& \geqslant d_{k-1}^{\mathrm{T}} y_{k-1}+d_{k-1}^{\mathrm{T}} d_{k-1}-d_{k-1}^{\mathrm{T}} y_{k-1} \\
& \geqslant d_{k-1}^{\mathrm{T}} d_{k-1} .
\end{aligned}
$$

It follows from (4) that

$$
\left\|d_{k}\right\| \geqslant\left(1-\frac{1}{4 t}\right)\left\|g_{k}\right\|
$$

then

$$
\alpha_{k} \leqslant \frac{\tau\left\|g_{k}\right\|}{\left\|d_{k}\right\|} \leqslant \frac{\tau}{1-\frac{1}{4 t}} \triangleq q .
$$

By (2), (7), and (8), it holds that

$$
\begin{aligned}
\left\|d_{k}\right\| \leqslant & \left\|g_{k}\right\|+\left(\frac{\left|g_{k}^{\mathrm{T}} y_{k-1}\right|}{d_{k-1}^{\mathrm{T}} \gamma_{k-1}}+\frac{t\left\|y_{k-1}\right\|^{2}\left|g_{k}^{\mathrm{T}} d_{k-1}\right|}{\left(d_{k-1}^{\mathrm{T}} \gamma_{k-1}\right)^{2}}\right)\left\|d_{k-1}\right\| \\
& \leqslant\left\|g_{k}\right\|+ \\
& \left(\frac{\alpha_{k-1} L\left\|g_{k}\right\|\left\|d_{k-1}\right\|}{\left\|d_{k-1}\right\|^{2}}+\frac{t \alpha_{k-1}^{2} L^{2}\left\|d_{k-1}\right\|^{2}\left\|g_{k}\right\|\left\|d_{k-1}\right\|}{\left\|d_{k-1}\right\|^{4}}\right)\left\|d_{k-1}\right\| \\
\leqslant & \left(1+\alpha_{k-1} L+t \alpha_{k-1}^{2} L^{2}\right)\left\|g_{k}\right\| \\
& \leqslant\left(1+q L+t q^{2} L^{2}\right)\left\|g_{k}\right\| .
\end{aligned}
$$

Since the level set $\Gamma$ is bounded and $g$ satisfies the Lipschitz condition, then the sequence $\left\{g_{k}\right\}$ is bounded. Hence the sequence $\left\{d_{k}\right\}$ is bounded. The remaining proof is referred to the second part of Theorem 3.1 in Ref. 16.

As pointed out by Cai et $\mathrm{al}^{14}$, if $\varphi_{\alpha}$ is convex, continuously differentiable, and first-order Lipschitz continuous, then $\mathscr{F}_{\alpha}$ is continuously differentiable and first-order Lipschitz continuous, i.e., $\nabla \mathscr{F}_{\alpha}$ is Lipschitz continuous. Thus if we select the appropriate $\varphi_{\alpha}(t)$, the problem (1) is solved by the MCG method. Applying the theorem to the function $\mathscr{F}_{\alpha}$, from the result in Ref. 14 we have the following global convergence results.

Theorem 2 Assume that $\varphi_{\alpha}(t)$ is even, convex, continuously differentiable, and strictly increasing in $|t|$. Let the sequence $\left\{u_{k}\right\}, k \geqslant 0$, be generated by the MCG method applied to $\mathscr{F}_{\alpha}$. If $\varphi_{\alpha}(t)$ is first-order Lipschitz continuous, then there exists a subsequence of $\left\{u_{k}\right\}$ converging to a global minimizer $u^{*}$ of $\mathscr{F}_{\alpha}$.

\section{NUMERICAL EXPERIMENTS}

In this section, some experimental results are given to show the performance of the MCG method in the context of impulse noise removal impulse noise. We select the salt-and-pepper impulse noise. The test images are $256 \times 256$ and $512 \times 512$ grey level images. We select the parameters in the MCG 
Table 1 Performance of the MCG and SP methods for restoring the images with noise level 50\%.

\begin{tabular}{lllrl}
\hline Image & Method & PSNR & Time $(\mathrm{s})$ & Iter. $^{\dagger}$ \\
\hline Lena & MCG $(\mathrm{t}=1)$ & 30.0952 & 2.7708 & 44.8 \\
$(256 \times 256)$ & MCG $(\mathrm{t}=2)$ & 30.1138 & 2.6402 & 44.4 \\
& SP & 29.9878 & 3.5500 & 44.6 \\
Cameraman & MCG $(\mathrm{t}=1)$ & 27.5288 & 3.7082 & 60.2 \\
$(256 \times 256)$ & MCG $(\mathrm{t}=2)$ & 27.5246 & 3.4110 & 59.4 \\
& SP & 27.5182 & 4.8000 & 61.0 \\
Barbara & MCG $(\mathrm{t}=1)$ & 26.4259 & 11.8686 & 41.4 \\
$(512 \times 512)$ & MCG $(\mathrm{t}=2)$ & 26.4213 & 10.9560 & 40.8 \\
& SP & 26.4032 & 16.4437 & 41.0 \\
Banoon & MCG $(\mathrm{t}=1)$ & 24.6085 & 12.0921 & 44.6 \\
$(512 \times 512)$ & MCG $(\mathrm{t}=2)$ & 24.5660 & 12.0572 & 44.6 \\
& SP & 24.5659 & 17.7937 & 45.0 \\
\hline
\end{tabular}

$\dagger$ Iter.,the total number of iterations for the whole impulse noise removal process.

Table 2 Performance of the MCG and SP methods for restoring the images with noise level $70 \%$.

\begin{tabular}{lllrl}
\hline Image & Method & PSNR & Time $(\mathrm{s})$ & Iter. \\
\hline Lena & MCG $(\mathrm{t}=1)$ & 27.0811 & 4.0648 & 63.0 \\
$(256 \times 256)$ & MCG $(\mathrm{t}=2)$ & 27.1862 & 4.0128 & 63.2 \\
& SP & 27.1024 & 5.2313 & 64.8 \\
Cameraman & MCG $(\mathrm{t}=1)$ & 24.6753 & 5.2796 & 81.4 \\
$(256 \times 256)$ & MCG $(\mathrm{t}=2)$ & 24.7377 & 5.0870 & 83.2 \\
& SP & 24.6262 & 6.7031 & 81.6 \\
Barbara & MCG $(\mathrm{t}=1)$ & 24.5696 & 17.2668 & 54.6 \\
$(512 \times 512)$ & MCG $(\mathrm{t}=2)$ & 24.5549 & 16.1910 & 53.6 \\
& SP & 24.5489 & 26.9312 & 63.4 \\
Banoon & MCG $(\mathrm{t}=1)$ & 22.3741 & 17.7564 & 59.4 \\
$(512 \times 512)$ & MCG $(\mathrm{t}=2)$ & 22.3729 & 17.9706 & 60.4 \\
& SP & 22.3724 & 25.9437 & 59.8 \\
\hline
\end{tabular}

Table 3 Performance of the MCG and SP methods for restoring the images with noise level $90 \%$.

\begin{tabular}{lllll}
\hline Image & Method & PSNR & Time $(\mathrm{s})$ & Iter. \\
\hline Lena & MCG $(\mathrm{t}=1)$ & 22.7776 & 10.8462 & 119.8 \\
$(256 \times 256)$ & MCG $(\mathrm{t}=2)$ & 22.7800 & 11.3956 & 135.2 \\
& SP & 22.7748 & 12.8906 & 123.0 \\
Cameraman & MCG $(\mathrm{t}=1)$ & 21.2164 & 12.6844 & 152.2 \\
$(256 \times 256)$ & MCG $(\mathrm{t}=2)$ & 21.2021 & 11.7630 & 150.0 \\
& SP & 21.1851 & 16.7437 & 147.4 \\
Barbara & MCG $(\mathrm{t}=1)$ & 22.5561 & 46.8472 & 105.8 \\
$(512 \times 512)$ & MCG $(\mathrm{t}=2)$ & 22.5594 & 44.0846 & 107.6 \\
& SP & 22.5512 & 62.1031 & 106.0 \\
Banoon & MCG $(\mathrm{t}=1)$ & 20.3252 & 42.4632 & 104.6 \\
$(512 \times 512)$ & MCG $(\mathrm{t}=2)$ & 20.3118 & 43.7204 & 106.0 \\
& SP & 20.3075 & 59.9218 & 107.8 \\
\hline
\end{tabular}

method as follows: $\delta=0.5, \tau=\sqrt{99} / 8, \rho=0.5$, $t=1,2, \varepsilon=10^{-4}$. In addition, we compare the performance of the MCG method with that of the SP method presented in Ref. 16. The SP method performs better than the Polak-Ribière conjugate gradient method which is the most effective among the methods considered in the numerical comparison in Ref. 14.

To solve the problem (1) by the MCG method, we select the Huber function ${ }^{18}$ as the edgepreserving function, which is convex and first-order Lipschitz continuous. Its definition is as follows

$$
\varphi_{\alpha}(t)= \begin{cases}\frac{t^{2}}{2 \alpha}, & |t| \leqslant \alpha, \\ |t|-\frac{\alpha}{2}, & |t|>\alpha,\end{cases}
$$

with $\alpha=10$. The stopping criterion is

$$
\frac{\left|F\left(u_{k}\right)-F\left(u_{k-1}\right)\right|}{\left|F\left(u_{k}\right)\right|} \leqslant 10^{-4} .
$$

To assess the restoration performance, we use the peak signal-to-noise ratio $\mathrm{PSNR}^{19}$, i.e.,

$$
\mathrm{PSNR}=10 \log _{10} \frac{255^{2}}{\frac{1}{M N} \sum_{i, j}\left(u_{i, j}^{*}-x_{i, j}\right)^{2}},
$$

where $u_{i, j}^{*}$ and $x_{i, j}$ denote the pixel values of the restored image and the original image, respectively. To test the performance of both methods more fairly, the experiments are repeated for 5 different noise samples of each image, and the average of the 5 results is listed in Tables $1-3$.

To a certain extent, Tables $1-3$ indicate that, for the PSNR, the MCG method performs better than the SP method for most of the test images with different noise levels. Although the numbers of iterations of both methods are very similar, the MCG method is faster than the SP method, leading to about 25\% saving in time. Fig. 1 lists the results restored by the methods from the corrupted images with noise level $70 \%$. In a word, these results show that the MCG method can effectively restore the corrupted images with different noise levels.

\section{CONCLUSIONS}

In this study, we propose a modified conjugate gradient method based on the HZ method. Remarkableness of the proposed method is that its global convergence is established under the Armijo line search without assuming that the objective is strongly convex. We use the proposed method to remove the impulse noise in the two-phase method, and obtain efficient experiment results. 

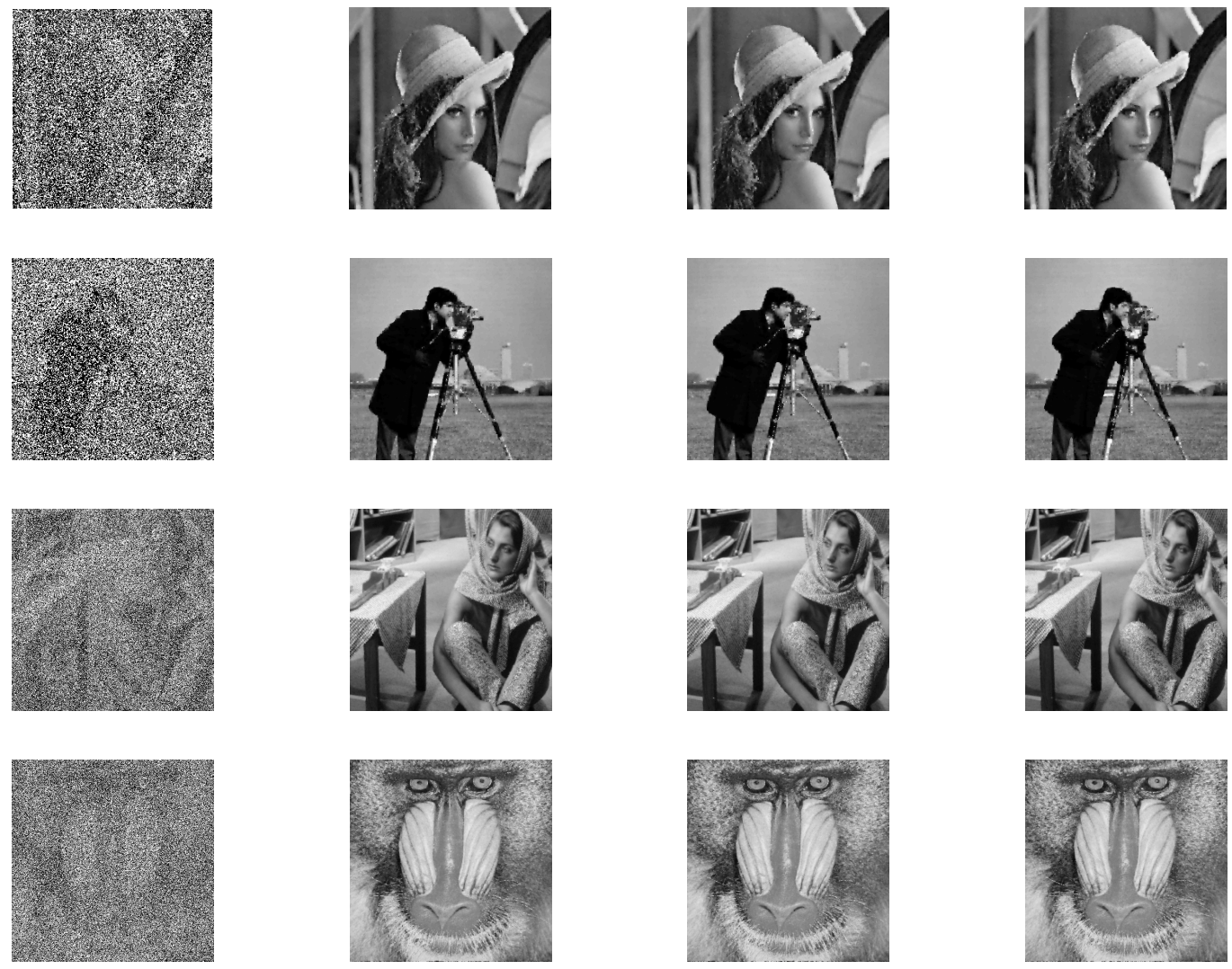

Fig. 1 Restoration of the images Lena, Cameraman, Barbara, and Banoon via the MCG $(t=1)$, MCG(t=2), and SP methods. From left to right: the corrupted image, the restorations obtained by the MCG(t=1), MCG(t=2), and SP methods, respectively.

\section{REFERENCES}

1. Hwang H, Haddad RA (1995) Adaptive median filters: new algorithms and results. IEEE Trans Image Process 4, 499-502.

2. Huang TS, Yang GJ, Tang GY (1979) A fast twodimensional median filtering algorithm. IEEE Trans Acoust Speech Signal Process 1, 13-8.

3. Astola J, Kuosmanen P (1997) Fundamentals of Nonlinear Digital Filtering, CRC, Boca Raton.

4. Nikolova M (2004) A variational approach to remove outliers and impulse noise. J Math Imaging Vision 20, 99-120.

5. Kimiaei M, Rostami M (2016) Impulse noise removal based on new hybrid conjugate gradient approach. Kybernetika 52, 791-823.

6. Veerakumar T, Jagannath RPK, Subudhi BN, Esakkirajan $S$ (2017) Impulse noise removal using adaptive radial basis function interpolation. Circ Syst Signal Process 36, 1192-223.

7. Veerakumar T, Subudhi BN, Esakkirajan S, Pradhan PK (2017) Context model based edge preservation filter for impulse noise removal. Expert Syst Appl 88,
29-44.

8. Chen QQ, Hung MH, Zou FM (2017) Effective and adaptive algorithm for pepper-and-salt noise removal. IET Image Process 11, 709-16.

9. Gupta V, Chaurasia V, Shandilya M (2015) Randomvalued impulse noise removal using adaptive dual threshold median filter. $J$ Vis Commun Image $R \mathbf{2 6}$, 296-304.

10. Esakkirajan S, Veerakumar T, Subramanyam AN, PremChand CH (2011) Removal of high density salt and pepper noise through modified decision based unsymmetric trimmed median filter. IEEE Signal Process Lett 18, 287-90.

11. Chan RH, Ho CW, Nikolova M (2005) Salt-andpepper noise removal by median-type noise detector and edge-preserving regularization. IEEE Trans Image Process 14, 1479-85.

12. Chen T, Wu HR (2001) Adaptive impulse detection using center-weighted median filters. IEEE Signal Process Lett 8, 1-3.

13. Chan RH, Ho CW, Leung CH, Nikolova M (2005) Minimization of detail-preserving regularization func- 
tional by Newton's method with continuation. In: Proceedings of IEEE International Conference on Image Processing, Genova, Italy.

14. Cai JF, Chan RH, Morini B (2007) Minimization of an edge-preserving regularization functional by conjugate gradient types methods. In: Tai XC, Lie KA, Chan TF, Osher S (eds) Image Processing Based on Partial Differential Equations, Spring, Berlin, pp 109-22.

15. Yu GH, Huang JH, Zhou Y (2010) A descent spectral conjugate gradient method for impulse noise removal. Appl Math Lett 23, 555-60.

16. Liu JK, Li SJ (2015) Spectral gradient method for impulse noise removal. Optim Lett 9, 1341-51.

17. Hager WW, Zhang H (2005) A new conjugate gradient method with guaranteed descent and an efficient line search. SIAM J Optim 16, 170-92.

18. Huber PJ (1973) Robust regression: Asymptotics, conjectures, and Monte Carlo. Ann Statist 1, 799-821.

19. Bovik A (2000) Handbook of Image and Video Processing, Academic, New York. 